\title{
Neutravidin-Mediated Extraction of Isolated Small Diameter Single Walled Carbon Nanotubes for Bio-Recognition
}

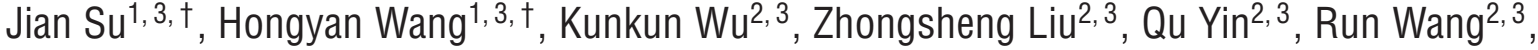 \\ Wei Lv², ${ }^{2}$, Shougen Yin ${ }^{1, *}$, Zunfeng $\operatorname{Liu}^{2,3, *}$, and Jan Pieter Abrahams ${ }^{4, *}$ \\ ${ }^{1}$ Key Laboratory of Display Materials and Photoelectric Devices, Ministry of Education, Institute of Material Physics, and \\ Tianjin Key Laboratory for Photoelectric Materials and Devices, Tianjin University of Technology, \\ Tianjin 300384, People's Republic of China \\ 2 Jiangsu Collaborative Innovation Center of Photovoltaic Science and Engineering, Department of Materials Science and Engineering, \\ Changzhou University, Changzhou 213164, China \\ 3 Jiangnan Graphene Research Institute, Changzhou 213149, China \\ ${ }^{4}$ C-CINA, Biozentrum Basel, CH-4003 Switzerland; LBR, Paul Scherrer Institute, Villigen, Switzerland
}

\begin{abstract}
Electron-transparent substrate that can specifically capture tagged protein complexes prompted us to fabricate a template that allow capture and structural studies of biotinylated protein complexes by transmission electron microscopy (TEM) to understand protein-protein interactions. Here we present a novel method that allows the scalable extraction and individualization of the tiny fraction of small diameter SWNTs that is functionalized with neutravidin, which can be used to bind biotinylated species. Atom force microscopy analysis indicates $98 \%$ purity of the individualized small diameter SWNTs (diameter range: $0.4-0.7 \mathrm{~nm}$, length range: $100-1000 \mathrm{~nm}$ ). The method includes two steps of functionalization-centrifugation based on the size selective properties of neutravidin toward the SWNTs and results in a sparsely neutravidin-functionalized population: about 1 biotin-specific neutravidin complex per $1000 \mathrm{~nm}$ of SNWT. The new method can be scaled up to mass production. This widely opens the door to the bulk characterization of its special (electronic) properties and will allow new, unprecedented applications of this fascinating material, such as electronic devices and novel biochemical applications. The SWNT-neutravidin could be used as a scaffold to analyze protein structure directly by cryo-transmission electron microscopy, which provides better understanding in protein-protein interactions and biological processes.
\end{abstract}

Keywords: Carbon Nanotubes, Purification, Protein, Bio-Recoginition.

\section{INTRODUCTION}

The need for an electron-transparent substrate that can specifically capture tagged protein complexes prompted us to fabricate small diameter single walled carbon nanotube (SWNT) functionalized with neutravidin. Such a template should allow structural studies of biotinylated protein or protein complexes by transmission electron microscopy (TEM) to understand protein-protein interactions. Single-particle cryo-EM is a well-known method for studying the $3 \mathrm{D}$ structures of protein complexes. ${ }^{1}$ Sample

\footnotetext{
*Authors to whom correspondence should be addressed.

${ }^{\dagger}$ These two authors contributed equally to this work.
}

preparation for cryo-EM is relatively straightforward, as it does not require a crystallization step (as for XRD) or a particularly large amount of sample (multi mg) at high concentrations (as for NMR spectroscopy). ${ }^{2}$ Proteincomplex purification is still a key step for the accurate characterization of the protein complex. Magnetic streptavidin beads efficiently capture biotinylated protein complexes using the extraordinarily high affinity constant of the streptavidin-biotin interaction. ${ }^{3}$ The target protein complex together with the magnetic beads can be quickly separated by the use of a magnet, but cannot be used directly for single-particle analysis via cryo-EM because the magnetic beads strongly interfere with the electron 
beam. In our pervious work, we have developed SWNT . streptavidin complexes and graphene oxide $\cdot$ streptavidin complexes, which can be used for affinity protein purification. Different from the magnetic beads, SWNT and graphene are almost electron transparent, therefore they can be directly used for structural characterization of the captured protein complexes after capturing of the protein purification. ${ }^{4,5}$ Therefore, such a purification process rolls the purification, sample preparation and characterization into a single step that can be handled quickly enough to retain the native structure of the protein complex. In our previous work, the most of the carbon nanotubes in SWNT - streptavidin complex are bundles, which is not ideal to be used as an electron transparent template. Therefore, to fabricate a bio-recognition system based on individualized small diameter carbon nanotube is highly demanded for obtain accurate 3D structures of the protein or protein complexes.

Single walled carbon nanotubes (SWNTs) with subnanometer diameter have promising properties, compared to large-diameter SWNTs because of the curvature effect. ${ }^{6}$ Sub-5 A SWNTs tend to be metallic due to strong $\Pi^{*}-\Pi^{*}$ hybridization effects which dramatically change the band structure, ${ }^{6}$ and $4 \AA$ SWNTs show superconductivity. ${ }^{7}$ Great efforts have been focused on creating small diameter SWNTs and their relative property research. The earliest report of the small diameter SWNTs goes back to $1992^{8}$ when individual $0.7 \mathrm{~nm}$ SWNTs were discovered in TEM studies of nanotubes produced by the arc-discharge method. SWNTs with diameters of $0.5,{ }^{9} 0.4,{ }^{10} 0.3^{11} \mathrm{~nm}$ were prepared in the central core of multiwalled carbon nanotubes, using the confinement provided by the outer graphite layer. A next major step forward was the preparation of uniformly sized small diameter SWNTs in the confined space of porous zeolite single crystal templates, ${ }^{12,13}$ making property investigations of the uniform small diameter SWNTs possible.7,14,15 The exciting progress is achieved by dissolving the zeolite with $\mathrm{HCl}$ to get the small diameter SWNTs without template. ${ }^{7,15,16}$ Furthermore, large-scale production of free and bundled small diameter SWNTs without template confinement has been realized, for instance by using catalytic chemical vapor deposition (CCVD) growth ${ }^{15}$ or the arc-discharge method. ${ }^{17}$ These small diameter SWNTs represent only a very small fraction of the bulk material, making it very difficult to study and explore their unique properties. This problem can be overcome by increasing the yield of small diameter SWNTs during the production stage, or by postproduction purification of small diameter SWNTs.

Great progress has been achieved in post-treatment of SWNTs, including purification, ${ }^{18-20}$ individualization, ${ }^{21-24}$ and fractionation. ${ }^{25-29}$ Soluble and individual SWNTs can be prepared through covalent functionalization ${ }^{24,30-32}$ and non-covalent 'molecular wrapping' with a surfactant, ${ }^{19,33-35}$ aromatic molecules, ${ }^{36-38}$ steroids, ${ }^{22}$ DNA,${ }^{20,39}$ phospholipids, ${ }^{40}$ peptides, ${ }^{41,42}$ and proteins. ${ }^{43-47}$
In general, individually dispersed nanotubes can be obtained by collecting the supernatant after ultracentrifugation, due to their relatively small density compared to bundles and impurities such as catalyst and carbon species. ${ }^{33}$ After solubilizing, diameter-selective fractionalization of SWNTs has been achieved by wrapping them with a third species such as SDS, ${ }^{48} \mathrm{DNA}^{20}$ or bile salts, ${ }^{25}$ followed by chromatography ${ }^{28,29,49}$ and density-gradient ultracentrifugation. ${ }^{25,48}$ Diameter-selective fractionalization can also be achieved during solubilization by repeated chemical functionalization $^{31}$ or specifically-designed aromatic polymers. ${ }^{26}$ Small diameter SWNTs produced by CVD and arc-discharge methods make up only a tiny fraction of all bulk material ${ }^{15,50}$ and always exist as bundles wrapped by a large amount of amorphous carbon. ${ }^{51}$ This makes extraction of small diameter SWNTs exceedingly difficult.

Protein molecules have been reported to scalably disperse SWNTs into separate tubes by noncovalent interaction. ${ }^{44,52}$ The debundling of SWNTs is attributed to the hydrophobic interaction between protein molecules and SWNTs. ${ }^{52}$ Furthermore, it was also reported that the interaction between carbon nanotubes and proteins depends greatly on the relative size of protein compared to the diameter of $\mathrm{CNT}^{52-55}$ i.e., protein molecules can more favorably bind to larger diameter CNTs which have small curvature surfaces. ${ }^{52}$ Consequently, it can be expected that protein molecules bind more strongly to SWNT-bundles than to individual large-diameter SWNTs, and only weakly bind to individual small diameter ones.

Proteins bound non-covalently to SWNTs are in equilibrium with proteins in solution and are expected to be released when dissolved protein is removed. As a result, SWNTs may aggregate and fall out of solution. ${ }^{44,45}$ One way of obtaining a stable conjugation of SWNTs with protein is to induce carbodiimidemediated crosslinking between the adsorbed proteins and SWNTs. ${ }^{45}$ This conjugation reaction occurs between the $\mathrm{COOH}$ groups of SWNT and the $-\mathrm{NH}_{2}$ groups in the protein molecule, resulting in a controlled solubilization of the SWNTs.

Oxidation creates COOH groups not only on SWNTs, but also on amorphous carbon impurities in the unfractionated SWNT sample which are more easily oxidized than the SWNTs, because carboxylic groups come from the intrinsic or induced defects and amorphous carbon has loose and non-crystalline structure and more defects compared to the SWNTs. ${ }^{56}$ This would imply that, after carbodiimide-mediated crosslinking to protein, the amorphous carbon should carry more proteins per surface area than the SWNTs.

Therefore, protein crosslinking could induce a higher solubility of amorphous carbon than of the individual large-diameter SWNT, while the sparsely functionalized individual small diameter SWNT should have the lowest solubility (see Fig. 1 for a schematic explanation). 


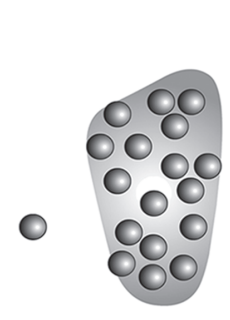

(a)

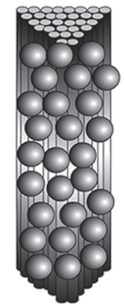

(b)

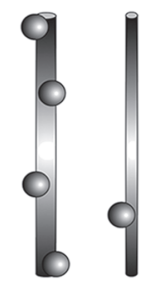

(d)

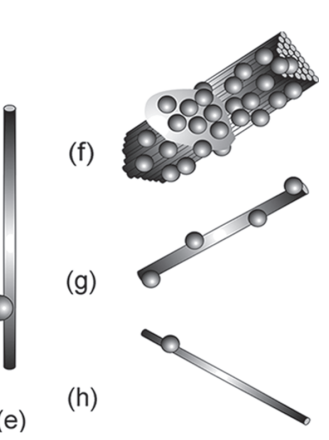

(e)

\section{RESULTS AND DISCUSSION}

\subsection{Purification of Small Diameter SWNT}

A commercial SWNT sample prepared by CVD was chosen as starting material. This material had been prepared according to the following procedure. First, $\mathrm{CH}_{4}$ is deposited on a cobalt-based catalyst, generating carbene and hydrogen. The carbene self-assembles into carbon nanotubes, releasing more hydrogen. Then the fresh-made SWNTs are treated with dilute nitric acid to remove the metal catalyst. The resulting preparation is oxidized by $\mathrm{KMnO}_{4}$, introducing $\mathrm{COOH}$ groups. The content of $\mathrm{COOH}$ is $2.7 \mathrm{wt} \%$. TEM characterization by the manufacturer indicated the diameter of the SWNTs to mainly be between 1-2 $\mathrm{nm}$. We found that the carboxylated SWNTs (SWNT-COOH) were insoluble in $\mathrm{H}_{2} \mathrm{O}$, forming aggregates and precipitating, SWNTs existed as bundles that were wrapped by amorphous carbon in aqueous solution, while individual SWNTs could hardly be seen, as shown in Figure 3(a).

We analyzed this commercial bulk material with Raman spectroscopy to establish the presence of the small diameter SWNTs (Fig. 2). After exciting at $785 \mathrm{~nm}$, a very weak peak could be detected in the radial breathing mode that extended from 506 to $560 \mathrm{~cm}^{-1}$ and was centered at $536 \mathrm{~cm}^{-1}$ (Fig. 2). This signal was caused by the minute fraction of small diameter SWNTs. From the Raman shiftdiameter relation $d(\mathrm{~nm})=224 / \omega\left(\mathrm{cm}^{-1}\right),{ }^{57}$ where $d$ is the diameter of individual SWNT and $\omega$ is the Raman frequency of the radial breathing mode, it could be deduced that these SWNTs had a small diameter around $0.4 \mathrm{~nm}$.

We used the procedure described by Yim et al. ${ }^{58}$ for carbodiimide-mediated crosslinking of neutravidin to the SWNTs (see Scheme 1). After the carbodiimide crosslinking of neutravidin, the SWNT-containing material could easily be dispersed in $\mathrm{H}_{2} \mathrm{O}$, in good agreement with the literature. ${ }^{45,58}$ Most SWNTs still existed as bundles wrapped by amorphous carbon, with neutravidin coated

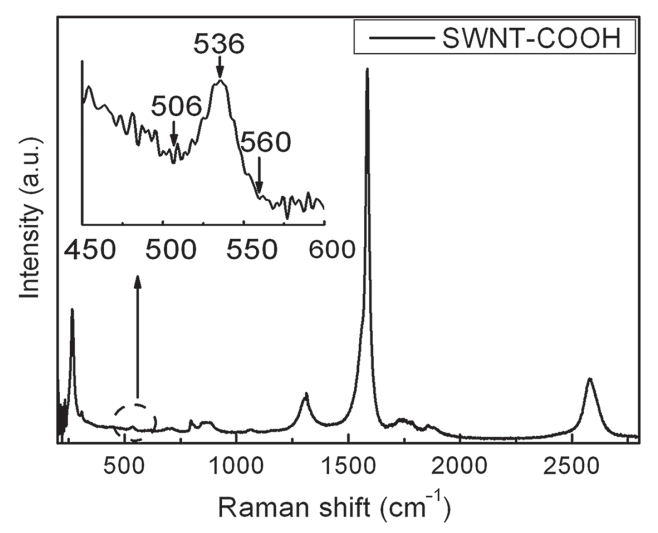

Figure 2. Raman spectrum of commercially available SWNT-COOH prepared by the CVD method, the weak peak at $536 \mathrm{~cm}^{-1}$ is shown in detail in the inset. From the relation between the frequency of RBM $\omega\left(\mathrm{cm}^{-1}\right)$ and the diameter of the nanotube $d(\mathrm{~nm}) \omega=224 / d$, it can be seen there are tiny amounts of small diameter SWNTs. 

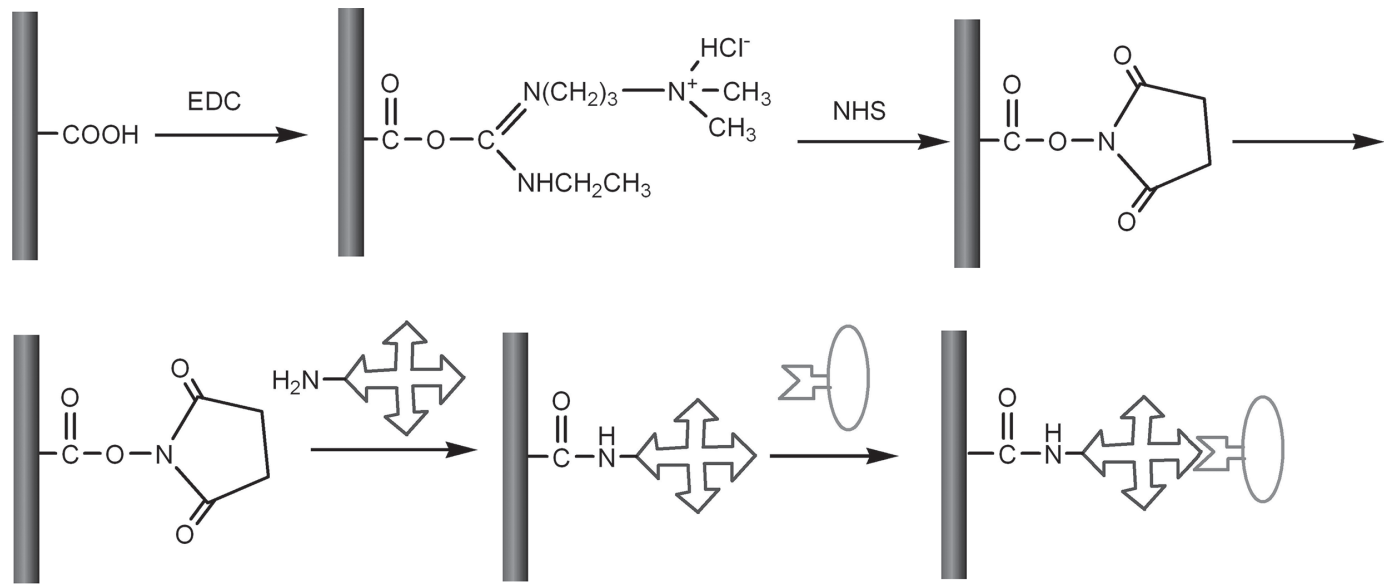

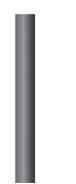
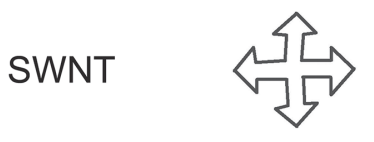

Neutravidin

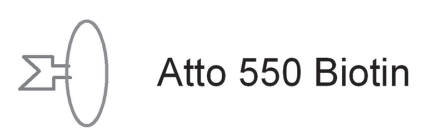

Scheme 1. Carbodiimide crosslinking of neutravidin with SWNT and the subsequent biotinylation with atto 550 Biotin as performed in this paper.

on them, see Figure 3(b). As stated above, we anticipated the amorphous carbon and the SWNT bundle to have a larger coverage of neutravidin molecules because of their relatively higher abundance of reactive $-\mathrm{COOH}$ and because of the preferred diameter selectivity of neutravidin molecules toward the SWNT bundles. ${ }^{52}$ Figure 3(c) shows a representative AFM image of a neutravidin coated SWNT bundle wrapped by amorphous carbon. It can be seen that the amorphous carbon indeed was virtually entirely covered with compactly-stacked neutravidin molecules, while there were also many neutravidin on the

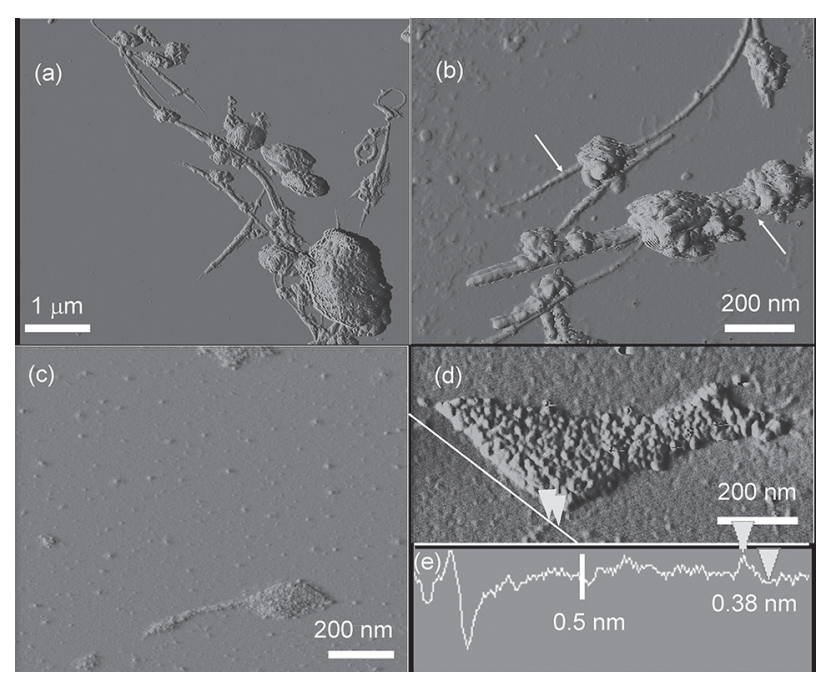

Figure 3. (a) AFM image of the SWNT-COOH, (b) AFM image of SWNTs after functionalization with neutravidin, the arrow indicates the neutravidin molecules, (c) AFM image of an SWNT bundle wrapped by amorphous carbon after functionalization with neutravidin, (d) AFM image of a small diameter SWNT extending out of an amorphous carbon after functionalized with neutravidin, (e) the cross-sectional analysis along the white line in image (d). surface of the SWNT bundle. Large-diameter SWNT bundles also had larger coverage of neutravidin compared to small ones, as indicated by the arrows in Figure 3(b). Individual $0.4 \mathrm{~nm}$ small diameter SWNTs occasionally stuck out of amorphous carbon, as shown in Figure 3(d), and were coated with relatively few neutravidin. Crosssectional analysis (Fig. 3(e)) indicated a diameter of $\sim 0.38 \mathrm{~nm}$, confirming the presence of small diameter SWNTs in the preparation.

The apparent differences in efficiency of covalent neutravidin binding between amorphous carbon, SWNT bundles, and the individual SWNTs, resulted in differences in solubility. The amorphous carbon and the SWNT bundles were most soluble, followed by the individual largediameter SWNTs, while the small diameter SNWTs were the least soluble. As a result, we could enrich the small diameter SWNTs by fractionated centrifugation. The best results were obtained by three times repeated centrifugation at $10000 \mathrm{rpm}$ for $20 \mathrm{~min}$, as shown in Scheme 2 . AFM confirmed that the final deposit (D) was enriched with SWNTs having the smallest diameter (Fig. 4(d)). The SWNTs were individually dispersed on the mica surface, but also some amorphous particles were observed. Cross-sectional analysis indicated that most of the SWNTs have diameters ranging from 0.4 to $0.7 \mathrm{~nm}$. The dispersion of the SWNTs (D) in water solution was not very stable, as they slowly precipitated over a period of days, while the supernatant solutions (A)-(C) remained stably dispersed for several weeks. Figures 4(a to c) show the AFM images of the supernatant solutions (A)-(C). As was expected, there were big bundles and amorphous carbon particles in the supernatants (A) and (B), due to their relatively good solubility. There were a large number of individually dispersed short SWNTs in the supernatant (A). 


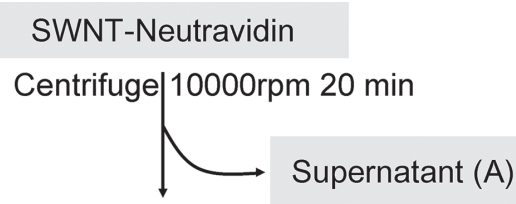

Deposit

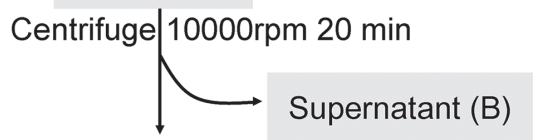

Deposit

Centrifuge|10000rpm $20 \mathrm{~min}$

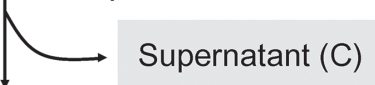

Deposit (D)

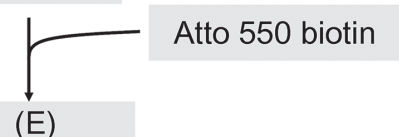
Centrifuge $\mid \begin{aligned} & 10000 \text { rpm } 20 \text { min } \\ & 3 \text { times }\end{aligned}$

Deposit (F)

Scheme 2. Post-treatment procedure after the carbodiimide crosslinking of neutravidin with SWNT and further functionalization with atto 550 biotin.

These individual SWNTs had a length below or around $200 \mathrm{~nm}$ and a diameter of around $1 \mathrm{~nm}$. These short individual SWNTs were enriched in the top layer of the supernatant after centrifugation, which was consistent with earlier observations that soluble individualized SWNTs can be prepared using surfactants such as SDS and SDBS. ${ }^{19,34}$ In these studies, the length of the individual SWNTs was always below $\sim 200 \mathrm{~nm}$. In supernatant (B), hardly any individual SWNTs were observed, while in supernatant (C), the individual SWNTs were present again, while the amount of SWNT bundles and amorphous carbon was much lower than in supernatant (A) and (B). The SWNTs in the supernatant (C) had diameter of $\sim 1 \mathrm{~nm}$ and length of 200-500 nm, as shown in Figure 4(e). In the deposit (D), we obtained sub-nm SWNTs having diameter of $0.4-0.7 \mathrm{~nm}$, as shown in Figure 4(f). We conclude that, besides extracting the individual small diameter SWNTs, the neutravidin functionalization and subsequent centrifugation also allows fractionation of SWNTs by length and diameter.

Although individual small diameter SWNTs could be extracted from the bulk material, the purification was not perfect, as there were still some amorphous carbon particles present in the deposit (D). Apparently, these amorphous carbon particles had similar solubility/density properties as the small diameter SWNTs, so that they could not be separated by centrifugation. The centrifugation

\section{2}
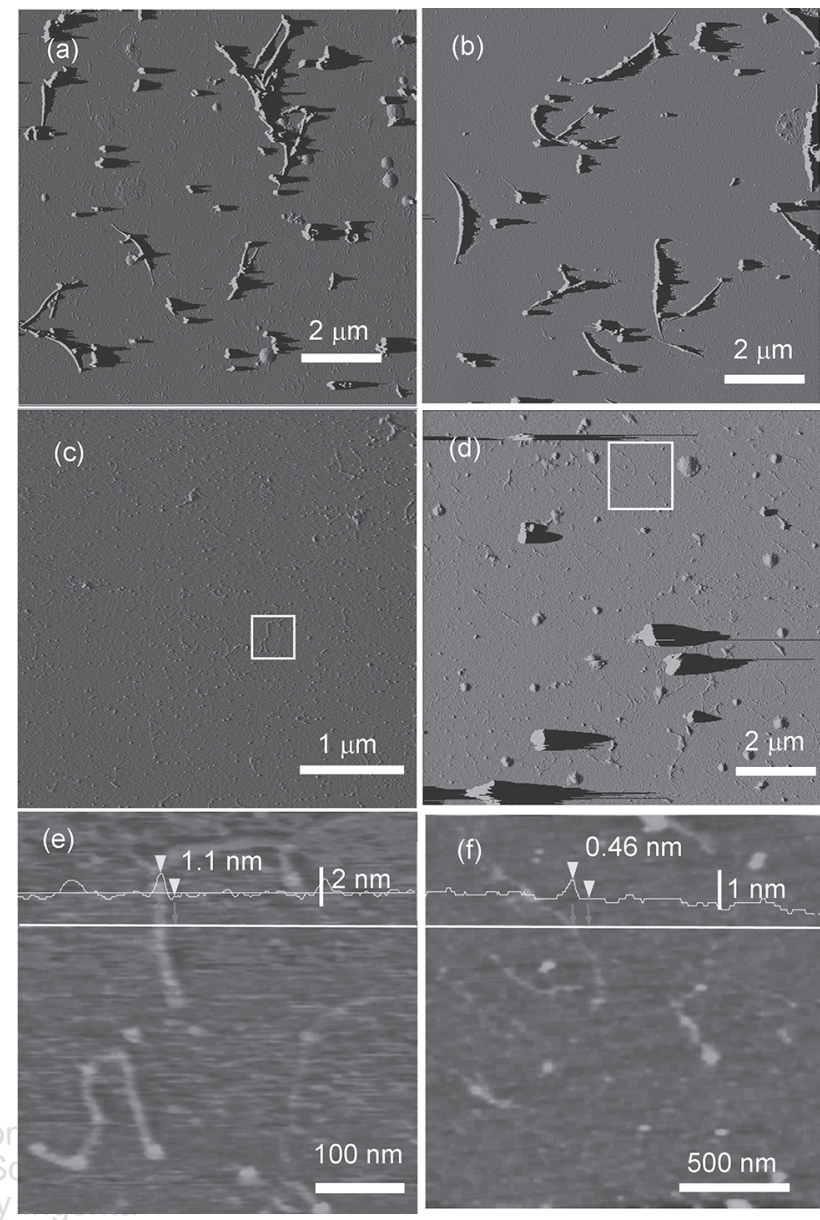

Figure 4. AFM images of supernatant (A), supernatant (B), supernatant (C) and the deposit (D) during the post-treatment of the SWNTs after carbodiimide crosslinking with neutravidin (see Scheme 2), are shown in (a-d), respectively; the area in squares in images (c and d) are magnified and shown in images (e and f).

speed and time were systematically varied to obtain optimal results.

Next, we performed an additional functionalization step, aimed to increase the solubility difference between the amorphous carbon particles and the small diameter SWNTs, as shown in Scheme 1. Here we used the biotinylated water-soluble fluorescent tag atto 550 biotin, which binds to SWNT-neutravidin due to the high affinity of neutravidin for the biotin moiety. Because neutravidin is a tetramer, four atto 550 biotin molecules can be bound per neutravidin complex. The solubility difference between the amorphous carbon and the individual small diameter SWNTs was hence expected to increase significantly. After this functionalization step, we could remove virtually all amorphous carbon by three times repeated fractional centrifugation (Scheme 2).

\subsection{AFM Analysis of Small Diameter SWNT}

The AFM image of the final fraction (F) of purified small diameter SWNTs is shown in Figure 5. Individual SWNTs 

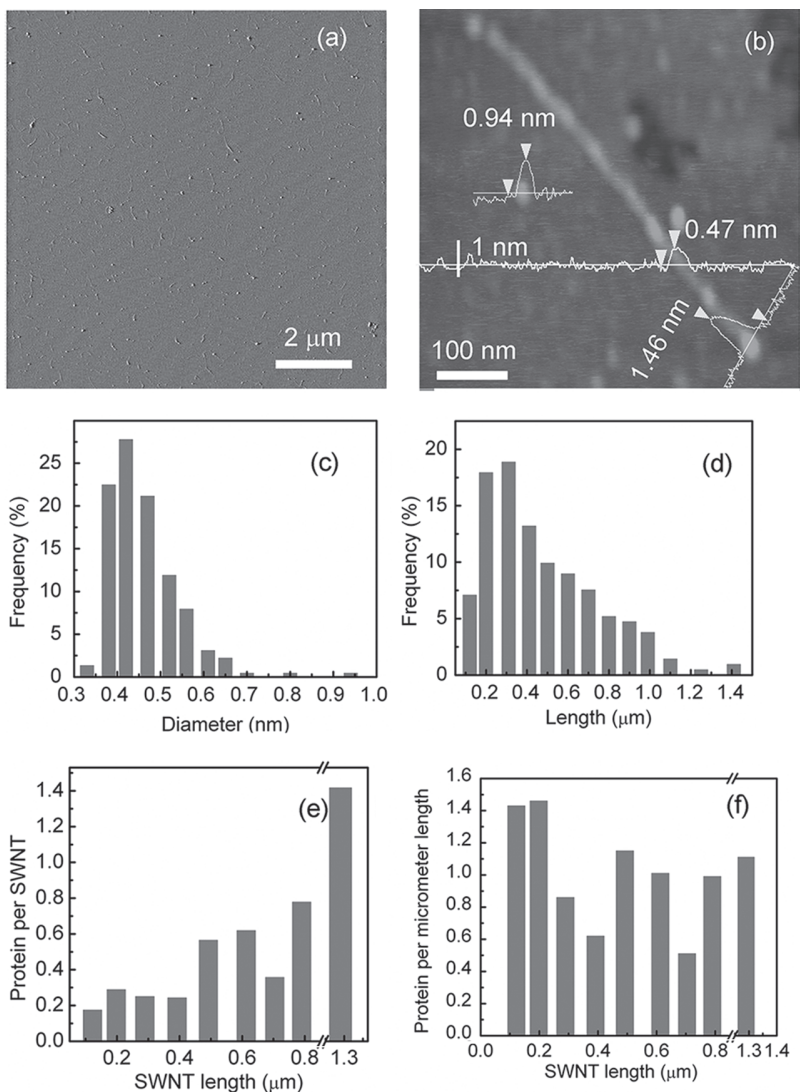

Figure 5. AFM image of SWNTs functionalized with neutravidin and then incubated with atto 550 biotin after repeated fractionation and centrifugation (deposit F, Scheme 2). A small magnification (a) shows the SWNTs were bent and a large magnification (b) allowed detailed measurements of diameter and neutravidin derivatisation. Diameter (c) and length (d) distribution of the SWNTs in deposit F after measuring 227 tubes; neutravidin number per SWNT as a function of SWNT length (e) and neutravidin number per micrometer length (f) in deposit F.

Note: Note that for reasons of clarity the $x$-axes of graphs (e and f) have been truncated.

with high purity dominated and almost all of the amorphous carbon particles had been removed from the SWNT preparations. Many SWNTs bent as curves on the mica surface, indicating a high flexibility (which confirms their small diameter). Figure 5(b) shows an AFM image at large magnification. The cross-sectional analysis indicated a diameter of $0.47 \mathrm{~nm}$ of this small diameter SWNT. In order to establish the distribution of length and diameter of the population of SWNTs, we measured 227 SWNTs using the AFM images (Figs. 5(c and d)). The diameter of the SWNTs is in the range of $0.4-0.7 \mathrm{~nm}$ by use of the cross-sectional analysis, and the length is in the range of 100-1000 nm, as shown in Figures 5(c and d).

We counted 708 individual SWNTs in the AFM images, accompanied by 11 amorphous carbon particles with sizes ranging from $50 \mathrm{~nm}$ to $100 \mathrm{~nm}$ and 3 SWNTs bundles with diameters ranging from 5 to $20 \mathrm{~nm}$ and a length of 0.3 to $2 \mu \mathrm{m}$. Based on these number the purity of the individualized small diameter SWNTs is calculated to be about $98 \%$. (Calculating: 708/(3+11+708) × 100\%) We also counted the number of neutravidin on the SWNTs and calculated their number per unit length of SWNTs. Neutravidin in solution is reported to be $\sim 60 \mathrm{kDa}$ a round particle with $\sim 5 \mathrm{~nm}$ diameter, but after drying, the neutravidin is anticipated to partially collapse. From dried neutravidin solution on mica, we observed by AFM that dried neutravidin particles had a height of $\sim 1 \mathrm{~nm}$. Figure $5(\mathrm{~b})$ shows a typical view of a dried neutravidin particle on mica substrate. Considering the typical small diameter of the SWNTs to be $\sim 0.4 \mathrm{~nm}$, the neutravidin molecule on SWNT should therefore be $\sim 1.4 \mathrm{~nm}$ in height. As shown in Figure 5(b) we can indeed observe a height of $1.46 \mathrm{~nm}$ for the neutravidin on an SWNT, indicating that we can observe single neutravidin bound to the nanotubes. By counting the neutravidin on 227 SWNTs, we estimated there were about 0.84 neutravidin per SWNT and $\sim 1$ neutravidin per micrometer length. The number of the neutravidin molecules attached to an SWNT increases with the increase of the tube length, as shown in Figure 5(e), but there is no significant effect of SWNT length on the number of neutravidin per unit length (Fig. 5(f)). There is $\sim 1$ neutravidin molecule per 1 micrometer length of SWNT. Please note that the average number of neutravidin molecules on the SWNTs (except for the very longest) is below 1, showing the weak interaction between the neutravidin molecule and the small diameter SWNTs. This implies that there must be SWNTs without neutravidin molecules on them, which may be due to the following two possible reasons.

(1) Not all of the neutravidin molecules are covalently bound to the SWNTs, on the contrary they physically absorb on SWNTs and dissociate from the SWNTs' wall during the repeated centrifugation and sonication stages.

(2) During the repeated sonication, some of the long SWNTs bearing neutravidin molecules were broken down to short tubes, and the SWNTs without neutravidin molecules were originally part of the long SWNTs.

In fact, we found that when leaving the solution of the fraction (F) for some time, part of the SWNTs would deposit in a few tens of minutes, while some would stay in solution for a couple of days. This was consistent with our finding that some SWNTs do not carry neutravidin on them, while the finding might also be explained by the possibility that neutravidin can dissociate from the SWNTs as time passes by.

Accurate measurements of the diameter of small diameter SWNTs have only been achieved by HR-TEM, ${ }^{15,59,60}$ but are still challenging, because of the great practical difficulty of accurately imaging narrow SWNTs. Due to radiation damage, they will fade under the electron beam within a few seconds, ${ }^{12}$ while it is difficult to correctly interpret the TEM images due to their weak scattering and the artifacts induced by interference effects at the defocusing conditions required to achieve sufficient contrast. ${ }^{61}$ On the other hand, AFM is a powerful, nondestructive 
technology for characterization of nanoscale and nanometer scale materials. By measuring the height, we can directly obtain the accurate diameter of the SWNTs- to our knowledge, up till now there was no report on successful diameter measurement of small diameter SWNTs using AFM. This may be because the content of the small diameter SWNT produced by the CVD or arc-discharge method is too low to allow their recognition. The first attempt to characterize small diameter SWNTs by AFM was carried out by Tang et al. on the SWNTs produced by zeolite template method. ${ }^{16}$ In Tang's study, the onedimensional feature (denotes the large aspect ratio) of the SWNTs could be observed, but an accurate measurement of their diameter was not achieved. Five possible reasons were given:

(1) the SWNTs tend to form aggregates and bundles and therefore individual SWNTs can hardly be observed;

(2) the SWNTs are not stable after they are extracted from the zeolite templates and break down into other graphite species and form clusters;

(3) most of the SWNTs are too short to be observed;

(4) the SWNTs do not adhere well enough to the substrate and may be pushed away from the scanned area;

(5) contaminations such as amorphous carbon from the precursor and possibly dissolved polymers from the container further complicate the observation of the small diameter SWNTs.

These problems would not only cause the failure of AFM diameter measurements, but would also impede other property measurements and applications.

Here, the AFM measurement of the small diameter SWNT could be carried out successfully and consistent values for their length and diameter could be obtained. The small diameter SWNTs did disperse individually on the mica substrate and they were stable during the whole post-treatment process, even under the sonication. Their length is within the range of 100-1000 $\mathrm{nm}$ and their clear one-dimensional, tubular structure allowed the accurate measurement of their diameter. The SWNTs were strongly attached on the mica substrate and no slippage was observed during the whole measurement. Furthermore, impurities, including the SWNTs bundles and amorphous carbon particles, could be removed to a large extent and the quality of obtained small diameter SWNTs allowed for highly-consistent AFM measurements.

After binding atto 550 to the SWNTs through the biotinneutravidin interaction, we also observed the SWNTs (deposit F) by fluorescence microscopy, the individual nanotubes could not be resolved in this experiment. These SWNTs show the strong, representative fluorescence signal of atto 550, while the supernatant of deposit (F) after the third centrifugation did not show any fluorescence signal under the same conditions. This indicated not only successful binding of atto 550 biotin to SWNT-neutravidin, but also showed that neutravidin retained its biotin-binding ability.

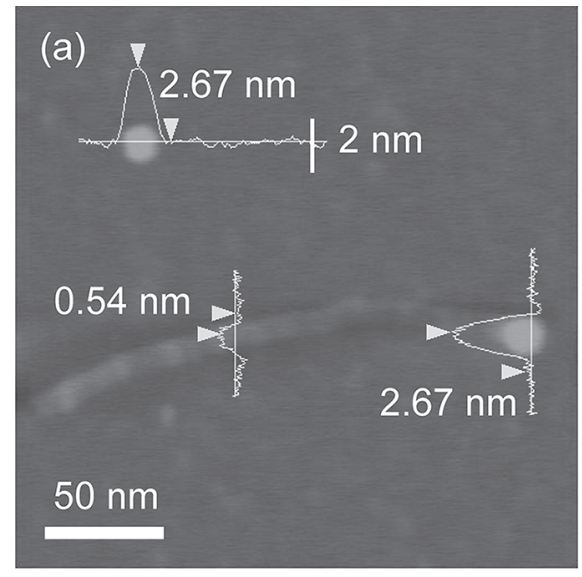

(b)

Figure 6. (a) AFM image of SWNT-Au nanoparticle complex linked by neutravidin-biotin interaction. (b) Schematic representation of relative position of SWNT and Au nanoparticle.

The strong adhesion of SWNTs to the mica substrate may be partly caused by their functionalization with neutravidin. After functionalization, the SWNTs anchored strongly on the mica substrate and did not slip during the repeated scanning with the AFM tip.

It has been reported that the streptavidin-biotin interaction can be reversibly broken in low salt at an elevated temperature. ${ }^{62}$ Assuming that the same would be true for neutravidin in view of its highly homologous biotin binding mechanism, we heated SWNT-neutravidin-biotin-atto 550 to $60{ }^{\circ} \mathrm{C}$ for $30 \mathrm{~s}$, and then replace the solvent with PBS buffer using a filter to remove the biotin-atto 550, and then mix with biotinylated gold nanoparticles (Au-biotin). If, at the elevated temperature, the atto 550 biotin dissociates from SWNT-neutravidin, there should be a mixture of SWNT-neutravidin, and atto 550 biotin in the solution. As the solution was replaced with PBS buffer, the biotinatto 500 will be removed, and SWNT-biotin-Au complexes should be formed after adding biotin-Au. Figure 6 shows an AFM image indicating that this indeed occurs. An Au nanoparticle with a diameter of $2.67 \mathrm{~nm}$ was linked to the end of an SWNT with a diameter of $0.54 \mathrm{~nm}$. Also in the image, a free Au nanoparticle with a diameter of $2.67 \mathrm{~nm}$ can be observed. This shows a possible application of the purified SWNTs: forming SWNT hybrids by neutravidinbiotin interaction.

\section{CONCLUSIONS}

In conclusion, a two-step functionalization-centrifugation protocol was used to extract and purify the small diameter SWNTs from commercially available bulk material containing SWNTs produced by a CVD method. In the first step, the neutravidin was covalently bound to the bulk material via the carbodiimide crosslinking 
and centrifugation allowed a substantial enrichment of the sample. After centrifugation the pellet contained mostly individualized small diameter SWNTs, with some amorphous carbon particles. In the second step, a water-soluble biotinylated fluorescence tag atto 550 biotin was used to further functionalize the SWNTs to increase the solubility difference between the SWNTs and the remaining amorphous carbon, followed by purification by repeated centrifugation. We showed by AFM that the diameter of these high-purity SWNTs is in the range of $0.4-0.7 \mathrm{~nm}$ and their length is in the range of 100-1000 nm. The small diameter SWNT-streptavidin complex could be used as a scaffold to analyze protein structure directly by cryo-transmission electron microscopy, which provides better understanding in protein-protein interactions and biological processes.

\section{EXPERIMENTAL SECTION}

Materials: SWNT-COOH (-COOH content: $2.7 \mathrm{wt} \%)$ prepared via a CVD method was purchased from Cheap Tubes Inc., SKU\# 0103. Neutravidin ${ }^{\mathrm{TM}}$ Biotin-binding protein (salt free) was purchased from Pierce Biotechnology. N-hydroxysuccinimide (NHS, 98\%), N-ethyl-N'-(3dimethylaminopropyl) carbodiimide hydrochloride (EDC, $98 \%)$, and atto 550 biotin $\left(1 \mathrm{mg} \mathrm{ml}^{-1}\right)$ were purchased from Sigma-Aldrich. Biotinylated Au nanoparticles (average diameter of $5 \mathrm{~nm}, 0.01 \%$ based on $\mathrm{Au}$ ) were purchased from NanoCS INC.

Reaction of SWNTs with Neutravidin and Purification: SWNT-COOH $(15.0 \mathrm{mg})$ was dispersed in MES buffer solution $(20 \mathrm{mM}, \mathrm{pH}=6.2,15 \mathrm{ml})$ through sonication with a tip sonicator (Sonics and Materials Inc. Model VC60) operating at $10 \mathrm{~W}$ (5 s on, $5 \mathrm{~s}$ off) for $30 \mathrm{~min}$.

Equal amounts $(2.5 \mathrm{ml})$ of the SWNT-COOH dispersion and a solution of $400 \mathrm{mM}$ NHS in MES buffer $(\mathrm{pH}=6.2)$ were mixed. This mixture was stirred for $30 \mathrm{~min}$ at $20^{\circ} \mathrm{C}$, followed by drop wise addition of EDC in MES buffer solution $(20 \mathrm{mM}, 5.0 \mathrm{ml})$ while stirring continuously. The mixture was stirred for another $30 \mathrm{~min}$, followed by membrane filtration (Millipore, GPWP04700, pore size: $0.45 \mu \mathrm{m}$, the same in the following experiments) and washing over a filter with MES buffer and then with PBS buffer in order to remove the excess NHS, EDC and byproducts. Then the activated SWNTs were dispersed in phosphate buffer saline $(10 \mathrm{ml})$ containing $\mathrm{KH}_{2} \mathrm{PO}_{4}$ $(5 \mathrm{mM}), \mathrm{K}_{2} \mathrm{H}_{2} \mathrm{PO}_{4}(15 \mathrm{mM}), \mathrm{NaCl}(150 \mathrm{mM})$ with $\mathrm{pH}=$ 7.1 , and mixed with neutravidin $\left(0.1 \mathrm{mg} \mathrm{ml}^{-1}\right)$ in the same phosphate buffer $(10 \mathrm{ml})$, followed by shaking at $200 \mathrm{rpm}$ for $3 \mathrm{~h}$ at $20^{\circ} \mathrm{C}$ in a refrigerated incubator/shaker (Innova ${ }^{\mathrm{TM}}$ 4330, New Brunswick Scientific).

The reaction product was membrane filtered and washed with $200 \mathrm{ml}$ of phosphate buffer, followed by $1000 \mathrm{ml}$ of PBS buffer. In order to thoroughly remove free neutravidin, these washing steps were repeated 3 times. SWNT-neutravidin was dispersed in $300 \mathrm{ml}$ of PBS buffer by sonication in a bath sonicator (Ultrasonic cleaner,
19 W, Model: 8848, Cole-Parmer) for $5 \mathrm{~min}$, centrifuged at $10,000 \mathrm{rpm}(10,392 \mathrm{~g})$ for 20 minutes in a laboratory centrifuge (Model: 3K18, Sigma Laboratory centrifuges, with rotor model $19776 \mathrm{H}$ ), and then filtered and washed with $1000 \mathrm{ml}$ of PBS buffer. The SWNT-neutravidin on membrane was re-dispersed in $100 \mathrm{ml}$ of milliQ $\mathrm{H}_{2} \mathrm{O}$ and centrifuged at 10,000 rpm for 20 minutes. The deposit was re-dispersed in $50 \mathrm{ml}$ of milliQ $\mathrm{H}_{2} \mathrm{O}$, and centrifuged at $10,000 \mathrm{rpm}$ for 20 minutes. This step was repeated this time dispersing the deposit in $30 \mathrm{ml}$ of $\mathrm{H}_{2} \mathrm{O}$ (solution A).

$0.1 \mathrm{ml}$ of atto 550 biotin solution $(0.01 \%$ according to $\mathrm{Au}$ ) was added to solution $\mathrm{A}$ followed by sonication using a tip sonicator at $10 \mathrm{~W}$ ( $5 \mathrm{~s}$ on, $5 \mathrm{~s}$ off $)$ for $5 \mathrm{~min}$. After sonication, the mixture was shaken at $200 \mathrm{rpm}$ for $1 \mathrm{~h}$ at $20{ }^{\circ} \mathrm{C}$, followed by tip sonication $(10 \mathrm{~W}, 5 \mathrm{~s}$ on, $5 \mathrm{~s}$ off) for $5 \mathrm{~min}$ and centrifuging at $10000 \mathrm{rpm}$ for $20 \mathrm{~min}$. The supernatant was discarded, $30 \mathrm{ml}$ of PBS buffer was added, followed by centrifugation at $10000 \mathrm{rpm}$ for $20 \mathrm{~min}$. The above step was repeated after addition of $10 \mathrm{ml}$ of PBS buffer and the final deposit was dispersed in $5 \mathrm{ml}$ of PBS buffer.

One $\mathrm{ml}$ of the final deposit was heated to $60{ }^{\circ} \mathrm{C}$ for $30 \mathrm{~s}$, and then the solution replaced with PBS buffer of $60{ }^{\circ} \mathrm{C}$ using a centrifuge filter, and re-dispersed in $1 \mathrm{ml}$ of PBS buffer. Gold nanoparticles were linked to the SWNT by mixing $0.1 \mathrm{ml}$ of biotinylated $\mathrm{Au}$ nanoparticles with $1 \mathrm{ml}$ of the above solution.

Measurements (AFM, and Raman): For atomic force microscopy (AFM), the sample solution was deposited onto a piece of pre-cleaved mica and measured after the solvent evaporated. Tapping mode was used to acquire the images under ambient conditions (AFM: Digital Instruments Dimension 3100).

Raman spectra were acquired on a calibrated Raman microscope setup (RamanRXN1 Microprobe, Kaiser optical Systems, Ann Arbor MI, USA) equipped with a diode laser at $785 \mathrm{~nm}$ operating at $23 \mathrm{~mW}$. Signals were taken from the solid carbon nanotube mixture at a spot size of $0.4 \mathrm{~mm}$ diameter, by summation of 100 scans of $1 \mathrm{~s}$.

Acknowledgments: This work was supported in China by the CMST no. 2013AA014201, NSFC (nos. 31200637, 61474082, 51303017), Jiangsu Basic Research Program grant BK2012148, Science and Technology Support Program of Changzhou grants CC20140016, CZ20140013, CJ20159037, CZ20150018, and CE20155017, Jiangsu Specially-Appointed Professor Program Sujiaoshi-2012-34.

\section{References and Notes}

1. R. Henderson, Quarterly Reviews of Biophysics 37, 3 (2004).

2. K. Asai, C. Sato, Y. Ueno, and K. Takahashi, Genome Informatics 11,151 (2000).

3. C. M. Niemeyer, Angew. Chem. Int. Ed. 40, 4128 (2001).

4. Z. Liu, L. Jiang, F. Galli, I. Nederlof, R. C. Olsthoorn, G. E. Lamers, T. Oosterkamp, and J. P. Abrahams, Adv. Funct. Mater. 20, 2857 (2010). 
5. Z. Liu, F. Galli, K. G. Janssen, L. Jiang, H. J. van der Linden, D. C. de Geus, P. Voskamp, M. E. Kuil, R. C. Olsthoorn, and T. H. Oosterkamp, The Journal of Physical Chemistry C 114, 4345 (2010).

6. X. Blase, L. X. Benedict, E. L. Shirley, and S. G. Louie, Phys. Rev. Lett. 72,1878 (1994).

7. Z. Tang, L. Zhang, N. Wang, X. Zhang, G. Wen, G. Li, J. Wang, C. Chan, and P. Sheng, Science 292, 2462 (2001).

8. P. Ajayan and S. Lijima, Nature 358, 23 (1992).

9. L. Sun, S. Xie, W. Liu, W. Zhou, Z. Liu, D. Tang, G. Wang, and L. Qian, Nature 403, 384 (2000).

10. L.-C. Qin, X. Zhao, K. Hirahara, Y. Miyamoto, Y. Ando, and S. Iijima, Nature 408, 50 (2000).

11. X. Zhao, Y. Liu, S. Inoue, T. Suzuki, R. Jones, and Y. Ando, Phys. Rev. Lett. 92, 125502 (2004).

12. N. Wang, Z.-K. Tang, G.-D. Li, and J. Chen, Nature 408, 50 (2000).

13. Z. Tang, H. Sun, J. Wang, J. Chen, and G. Li, Appl. Phys. Lett. 73, 2287 (1998).

14. Z.-K. Tang, N. Wang, X. Zhang, J. Wang, C.-T. Chan, and P. Sheng, New Journal of Physics 5, 146 (2003).

15. T. Hayashi, Y. A. Kim, T. Matoba, M. Esaka, K. Nishimura, T. Tsukada, M. Endo, and M. S. Dresselhaus, Nano Lett. 3, 887 (2003).

16. X. Zhang, J. Ye, H. Yang, C. Zhang, K. M. Ho, T. Su, N. Wang, Z. Tang, and X. Xiao, Surf. Rev. Lett. 14, 687 (2007).

17. Y. Li, S. Xie, W. Zhou, D. Tang, Z. Liu, X. Zou, and G. Wang, Carbon 39, 1429 (2001).

18. A. Ikeda, Y. Tanaka, K. Nobusawa, and J.-I. Kikuchi, Langmuir 23, 10913 (2007).

19. Z. Sun, V. Nicolosi, D. Rickard, S. D. Bergin, D. Aherne, and J. N. Coleman, The Journal of Physical Chemistry C 112, 10692 (2008).

20. M. Zheng, A. Jagota, E. D. Semke, B. A. Diner, R. S. McLean, S. R. Lustig, R. E. Richardson, and N. G. Tassi, Nat. Mater. 2, 338 (2003).

21. T. Hasan, V. Scardaci, P. Tan, A. G. Rozhin, W. I. Milne, and A. C. Ferrari, The Journal of Physical Chemistry C 111, 12594 (2007).

22. A. Ishibashi and N. Nakashima, Chem. Eur. J. 12, 7595 (2006).

23. T. H. Kim, C. Doe, S. R. Kline, and S. M. Choi, Adv. Mater. 19, 929 (2007)

24. N. I. Kovtyukhova, T. E. Mallouk, L. Pan, and E. C. Dickey, J. Am. Chem. Soc. 125, 9761 (2003)

25. M. S. Arnold, A. A. Green, J. F. Hulvat, S. I. Stupp, and M. C. Hersam, Nat. Nanotechnol. 1, 60 (2006).

26. F. Chen, B. Wang, Y. Chen, and L.-J. Li, Nano Lett. 7, 3013 (2007).

27. Z. Chen, X. Du, M.-H. Du, C. D. Rancken, H.-P. Cheng, and A. G. Rinzler, Nano Lett. 3, 1245 (2003).

28. D. A. Heller, R. M. Mayrhofer, S. Baik, Y. V. Grinkova, M. L. Usrey, and M. S. Strano, J. Am. Chem. Soc. 126, 14567 (2004)

29. A. A. Vetcher, S. Srinivasan, I. A. Vetcher, S. M. Abramov, M. Kozlov, R. H. Baughman, and S. D. Levene, Nanotechnology 17, 4263 (2006).

30. C. A. Dyke and J. M. Tour, Nano Lett. 3, 1215 (2003).

31. K. S. Fernando, Y. Lin, W. Wang, L. Cao, M. J. Meziani, X. Wang, M. L. Veca, P. Zhang, R. A. Quinn, and L. F. Allard, The Journal of Physical Chemistry C 111, 10254 (2007).

32. G. Viswanathan, N. Chakrapani, H. Yang, B. Wei, H. Chung, K. Cho, C. Y. Ryu, and P. M. Ajayan, J. Am. Chem. Soc. 125, 9258 (2003).

33. M. J. O'connell, S. M. Bachilo, C. B. Huffman, V. C. Moore, M. S. Strano, E. H. Haroz, K. L. Rialon, P. J. Boul, W. H. Noon, and C. Kittrell, Science 297, 593 (2002).
34. J. Y. Shin, T. Premkumar, and K. E. Geckeler, Chem. Eur. J. 14, 6044 (2008).

35. W. Wenseleers, I. I. Vlasov, E. Goovaerts, E. D. Obraztsova, A. S. Lobach, and A. Bouwen, Adv. Funct. Mater. 14, 1105 (2004).

36. J. Chen and C. P. Collier, The Journal of Physical Chemistry B 109, 7605 (2005)

37. J.-Y. Hwang, A. Nish, J. Doig, S. Douven, C.-W. Chen, L.-C. Chen, and R. J. Nicholas, J. Am. Chem. Soc. 130, 3543 (2008).

38. A. Nish, J.-Y. Hwang, J. Doig, and R. J. Nicholas, Nat. Nanotechnol. 2, 640 (2007).

39. S. E. Snyder and S. V. Rotkin, Small 4, 1284 (2008).

40. Y. Wu, J. S. Hudson, Q. Lu, J. M. Moore, A. S. Mount, A. M. Rao, E. Alexov, and P. C. Ke, The Journal of Physical Chemistry B 110, 2475 (2006)

41. L. S. Witus, J.-D. R. Rocha, V. M. Yuwono, S. E. Paramonov, R. B. Weisman, and J. D. Hartgerink, J. Mater. Chem. 17, 1909 (2007).

42. G. R. Dieckmann, A. B. Dalton, P. A. Johnson, J. Razal, J. Chen, G. M. Giordano, E. Muñoz, I. H. Musselman, R. H. Baughman, and R. K. Draper, J. Am. Chem. Soc. 125, 1770 (2003).

43. P. Goldberg-Oppenheimer and O. Regev, Small 3, 1894 (2007).

44. S. S. Karajanagi, H. Yang, P. Asuri, E. Sellitto, J. S. Dordick, and R. S. Kane, Langmuir 22, 1392 (2006).

45. Y. Lin, L. F. Allard, and Y.-P. Sun, The Journal of Physical Chemistry $B$ 108, 3760 (2004).

46. K. Matsuura, T. Saito, T. Okazaki, S. Ohshima, M. Yumura, and S. Iijima, Chem. Phys. Lett. 429, 497 (2006).

47. D. Nepal and K. E. Geckeler, Small 3, 1259 (2007).

48. A. A. Green and M. C. Hersam, Nano Lett. 8, 1417 (2008).

49. M. Zheng, A. Jagota, M. S. Strano, A. P. Santos, P. Barone, S. G. Chou, B. A. Diner, M. S. Dresselhaus, R. S. Mclean, and G. B. Onoa, Science 302, 1545 (2003).

50. C. Marın, M. Serrano, N. Yao, and A. Ostrogorsky, Nanotechnology 14, L4 (2003). 14:28:38

51. Y. Lin, D. Hill, J. Bentley, L. Allard, and Y.-P. Sun, Microsc. Microanal. 9, 352 (2003)

52. Q. Mu, W. Liu, Y. Xing, H. Zhou, Z. Li, Y. Zhang, L. Ji, F. Wang, Z. Si, and B. Zhang, The Journal of Physical Chemistry C 112, 3300 (2008).

53. N. W. S. Kam and H. Dai, J. Am. Chem. Soc. 127, 6021 (2005)

54. E. Katz and I. Willner, ChemPhysChem. 5, 1084 (2004).

55. C. Salvador-Morales, E. Flahaut, E. Sim, J. Sloan, M. L. Green, and R. B. Sim, Molecular Immunology 43, 193 (2006)

56. Y.-P. Sun, K. Fu, Y. Lin, and W. Huang, Acc. Chem. Res. 35, 1096 (2002).

57. S. Bandow, S. Asaka, Y. Saito, A. Rao, L. Grigorian, E. Richter, and P. Eklund, Phys. Rev. Lett. 80, 3779 (1998).

58. T.-J. Yim, J. Liu, Y. Lu, R. S. Kane, and J. S. Dordick, J. Am. Chem. Soc. 127, 12200 (2005).

59. L.-C. Qin, X. Zhao, K. Hirahara, Y. Ando, and S. Iijima, Chem. Phys. Lett. 349, 389 (2001).

60. Y. Chan, H. Peng, Z. Tang, and N. Wang, Chem. Phys. Lett. 369, 541 (2003).

61. T. Hayashi, H. Muramatsu, Y. A. Kim, H. Kajitani, S. Imai, H. Kawakami, M. Kobayashi, T. Matoba, M. Endo, and M. S. Dresselhaus, Carbon 44, 1130 (2006)

62. O. Nord, M. Lukacs, J. Lundeberg, and M. Uhlén, Electrophoresis 26, 501 (2005)

63. Cheap Tubes Inc., http://www.CheapTubes.com (2008), SKU\#: 0103

Received: 13 January 2016. Accepted: 2 March 2016. 